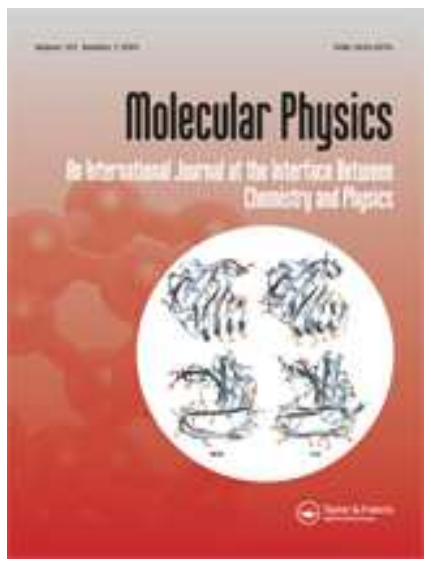

\title{
Fluid of disks with competing interactions
}

\begin{tabular}{|c|c|}
\hline Journal: & Molecular Physics \\
\hline Manuscript ID: & TMPH-2011-0227.R1 \\
\hline Manuscript Type: & Special Issue in honour of Luciano Reatto \\
\hline $\begin{array}{l}\text { Date Submitted by the } \\
\text { Author: }\end{array}$ & 09-Aug-2011 \\
\hline Complete List of Authors: & Roth, Roland; University of Erlangen \\
\hline Keywords: & $\begin{array}{l}\text { Density Functional Theory, competing interaction, cluster formation, } \\
\text { lamellar structure }\end{array}$ \\
\hline \multicolumn{2}{|c|}{$\begin{array}{l}\text { Note: The following files were submitted by the author for peer review, but cannot be converted } \\
\text { to PDF. You must view these files (e.g. movies) online. }\end{array}$} \\
\hline
\end{tabular}

\section{SCHOLARONE ${ }^{\text {Im }}$ Manuscripts}


Molecular Physics

Vol. 00, No. 00, Month 200x, 1-12

\title{
RESEARCH ARTICLE
}

\section{Fluid of disks with competing interactions}

\author{
Roland $\operatorname{Roth}^{a}$ \\ ${ }^{a}$ Institut für Theoretische Physik, Universität Erlangen-Nürnberg, Staudtstr. 7, 91058 \\ Erlangen, Germany \\ (Received 00 Month 200x; final version received 00 Month 200x)
}

\begin{abstract}
We study within the framework of density functional theory the behaviour of a two dimensional fluid with a competing attraction at short distances and repulsion at longer distance in addition to a hard-core. The soft part of the interaction is treated by a first-order thermodynamic perturbation theory, which for a homogeneous bulk fluid predicts that thermodynamic quantities reduce to that of the hard-disk reference system. Despite this seemingly simple thermodynamic result, the behaviour of this fluid with competing interactions is rich and intriguing. In this work we follow the insight and inspirations by Reatto and co-workers, who performed computer simulation studies of the same system.
\end{abstract}

\section{Introduction}

A variety of interesting fluid behaviour can be observed in model systems that interact via pair potentials with strong, hard-core repulsion at short distances and a soft attraction $[1]$

$$
V(r)=\left\{\begin{array}{cl}
\infty & r<2 R \\
V_{\text {soft }}(r) & \text { otherwise }
\end{array}\right.
$$

Here $R$ is the radius of the hard-core interaction. In the case of simple liquids, where the soft part of the interaction is a purely attractive potential, phase separation into a low density gas and a high density liquid can be found at sufficiently low temperatures. Simple fluids are, of course, well studied in theory and experiment.

Very different behaviour follows if the soft interaction is given by competing attraction at short distances and a longer ranged repulsion of the form $[2-7]$

$$
V_{\text {soft }}(r)=-\frac{\varepsilon_{a} \sigma^{2}}{R_{a}^{2}} \exp \left(-\frac{r}{R_{a}}\right)+\frac{\varepsilon_{r} \sigma^{2}}{R_{r}^{2}} \exp \left(-\frac{r}{R_{r}}\right)
$$

with $\varepsilon_{a}>0$ and $\varepsilon_{r}>0$, and $R_{a}<R_{r}$. The hard-core diameter of the disk $\sigma=2 R$. This potential was first introduced by Sear et al. [2,3] to study the formation of clusters, or micro-phase separation, of nanoparticles (quantum dots) trapped at a water-air interface. That rich and interesting equilibrium behaviour should occur was recognised and studied by Reatto and co-workers. The model given by Eq. (2) in dimension $d=2$ was studied by Imperio and Reatto [4-6]. A slightly modified version of this model in $d=3$, where the exponential functions were replaced by Yukawa interactions, was studied Reatto and colleagues [8-10] and also inspired studies by Archer et al. [11, 12] and by Li et al. [13].

Here we study, inspired by Reatto's insights and findings, the two dimensional system with pair interactions given by Eq. (2) within the framework of classical 
density functional theory (DFT). By doing so we hope to add a somewhat different perspective to the results already reported on this intriguing system. In particular we make use of the fact that within DFT it is possible to access the structure and the thermodynamics of a system on equal footing.

The paper is organised as follows. In Section 2 we describe the theoretical framework of this study. The main ideas of DFT are outlined for the particular application. The system of disks with competing interactions is studied first in the absence of any external field in Section 3. In Section 4 a single planar hard wall is added to the system. One point of particular interest here is the fact that the wall contact theorem imposes a constraint on the density profile. In Section 5 we add a second parallel hard wall and study the disks in a simple confined geometry. We conclude with a discussion of our results in Section 6.

\section{Density Functional Theory}

Within the framework of density functional theory for classical systems [14, 15] there exists a functional of the average one-body density $\rho(\mathbf{r})$ which upon minimisation yields the grand canonical potential:

$$
\Omega[\rho]=\mathcal{F}[\rho]+\int d^{2} r \rho(\mathbf{r})\left(V_{\text {ext }}(\mathbf{r})-\mu\right),
$$

where $\mathcal{F}[\rho]$ is the intrinsic Helmholtz free energy functional, $V_{\text {ext }}(\mathbf{r})$ is the external and $\mu$ the chemical potential.

The intrinsic Helmholtz free energy functional can be split into the exact ideal gas part

$$
\mathcal{F}_{i d}[\rho]=\beta^{-1} \int d^{2} r \rho(\mathbf{r})\left(\ln \lambda^{2} \rho(\mathbf{r})-1\right)
$$

where this is written in dimension $d=2$ and an excess (over the ideal gas) free energy $\mathcal{F}_{\text {ex }}[\rho]$

$$
\mathcal{F}[\rho]=\mathcal{F}_{i d}[\rho]+\mathcal{F}_{\text {ex }}[\rho] .
$$

$\lambda$ is the thermal wavelength. The excess free energy contains all the information about the inter-particle interactions and for most systems of interest is only known approximately. Once the functional of the excess free energy is constructed it can be employed to study the system in an arbitrary external field described by $V_{\text {ext }}(\mathbf{r})$. By minimising the functional of the grand potential, Eq. (3), one can obtain the generally inhomogeneous equilibrium structure, $\rho_{0}(\mathbf{r})$, and the thermodynamics of the system through the relation $\Omega=\Omega\left[\rho_{0}(\mathbf{r})\right]$, where $\Omega$ is the grand potential of the system. In the following we will suppress the index 0 , that indicates the fact that an equilibrium structure is used.

Since the excess free energy functional for a system of disks interacting with competing interactions is not known we follow a standard perturbation theory approach in which we use a fluid of hard-disks $(H D)$ as reference system $[16,17]$ described by fundamental-measure theory (FMT) $[18,19]$ and a perturbation term for the soft interaction:

$$
\mathcal{F}_{\text {ex }}[\rho]=\mathcal{F}_{\text {ex }}^{H D}[\rho]+\frac{1}{2} \int d^{2} r \rho(\mathbf{r}) \int d^{2} r^{\prime} \rho\left(\mathbf{r}^{\prime}\right) \tilde{V}_{\text {soft }}\left(\left|\mathbf{r}-\mathbf{r}^{\prime}\right|\right) .
$$


In FMT the excess free energy of a hard-core system is written as $[18,19]$

$$
\beta \mathcal{F}_{\text {ex }}[\rho]=\int d^{2} r \phi\left(n_{\alpha}\right)
$$

where the excess free energy density $\phi$ is a function of weighted densities $n_{\alpha}$. The form of $\phi$ used here and the weight functions are given in Ref. [17].

A first order perturbation theory of the form (6) is known to underestimate the correlation in the system. Therefore the soft interaction part in the pair potential (2), $V_{\text {soft }}(r)$, is empirically changed to $\tilde{V}_{\text {soft }}(r)$ in Eq. (6), and is defined by

$$
\beta \tilde{V}_{\text {soft }}(r)=\left\{\begin{array}{cc}
-\beta V_{\text {soft }}(r=2 R) & r<2 R \\
\beta V_{\text {soft }}(r) & \text { otherwise }
\end{array}\right.
$$

where the attraction is extended into the hard core, i.e. to $r \rightarrow 0$. As usual, $\beta=$ $1 /\left(k_{B} T\right)$ with Boltzmann constant $k_{B}$ and absolute temperature $T$.

Following Imperio and Reatto $[4,5]$ the length scales $R_{a}$ and $R_{r}$ in the soft interaction, Eq. (2), are set to $R_{a}=2 R$ and $R_{r}=4 R$. In addition a relation between the depth of the attraction $\varepsilon_{a}$ and the strength of the repulsion $\varepsilon_{r}$ is established by imposing the condition that

$$
\int d^{2} r \tilde{V}_{s o f t}(r)=0
$$

This is slightly different from the condition imposed on the interaction potential $V_{\text {soft }}(r)$ within simulations by Imperio and Reatto $[4,5]$ where $\int d^{2} r V_{\text {soft }}(r)=0$ leads to $\varepsilon_{a}=\varepsilon_{r}$. Here, the condition (9) leads for the present choice of length scales to

$$
\varepsilon_{r}=\frac{20}{13 \sqrt{e}} \varepsilon_{a} \approx 0.933124 \varepsilon_{a}
$$

where $e$ is the Euler constant.

This concludes the description of the density functional. The first application of the functional is to a bulk fluid, i.e. to a system without an external potential.

\section{Bulk Fluid}

Within first order thermodynamic perturbation theory, which corresponds to the approximate treatment of the soft interaction within DFT given by Eq. (6), the equation of state and the chemical potential of a homogeneous bulk fluid can be calculated straightforwardly from the excess Helmholtz free energy density (in $d=2$ ) $f_{\text {ex }}=F_{\text {ex }} / A=\mathcal{F}_{\text {ex }}\left[\rho(\mathbf{r})=\rho_{\text {bulk }}\right] / A$ with the constant bulk density $\rho_{\text {bulk }}$. Note that in the absence of an external potential, simple fluids exhibit in a homogeneous bulk system, in the region of density and temperature away from crystallisation. This is not necessarily true for a system with competing interaction. However, if we start by considering sufficiently high temperatures and low densities, a homogeneous bulk fluid can be assumed also for the present system.

It is easy to see from Eq. (6) that

$$
f_{e x}\left(\rho_{\text {bulk }}\right)=f_{\text {ex }}^{H D}\left(\rho_{\text {bulk }}\right)+\frac{1}{2} \rho_{\text {bulk }}^{2} \int d^{2} r \tilde{V}_{\text {soft }}(r)=f_{\text {ex }}^{H D}\left(\rho_{\text {bulk }}\right),
$$


where we have employed condition (9) in the second step. Hence for a homogeneous bulk fluid the excess free energy density of a system with competing interactions reduces to that of the hard-disks reference system. As a consequence of (11) fixed by (9) also the equation of state, $p$, and the chemical potential, $\mu$, of a homogenous system of disks with competing interactions reduces exactly to those of hard disks. The equation of state underlying the FMT $[16,17]$ functional employed here is that of scaled-particle theory (SPT) in two dimensions

$$
\beta p^{H D}=\beta p^{S P T}=\frac{\rho}{(1-\eta)^{2}}
$$

where the packing fraction $\eta=\rho_{b u l k} R^{2} \pi$. This is known to account accurately (compared to computer simulations) for the pressure of a hard-disk fluid. Thus the grand potential $\Omega$ of a homogeneous bulk system is the same as that of hard-disk reference fluid

$$
\Omega=-p A=-p^{H D} A
$$
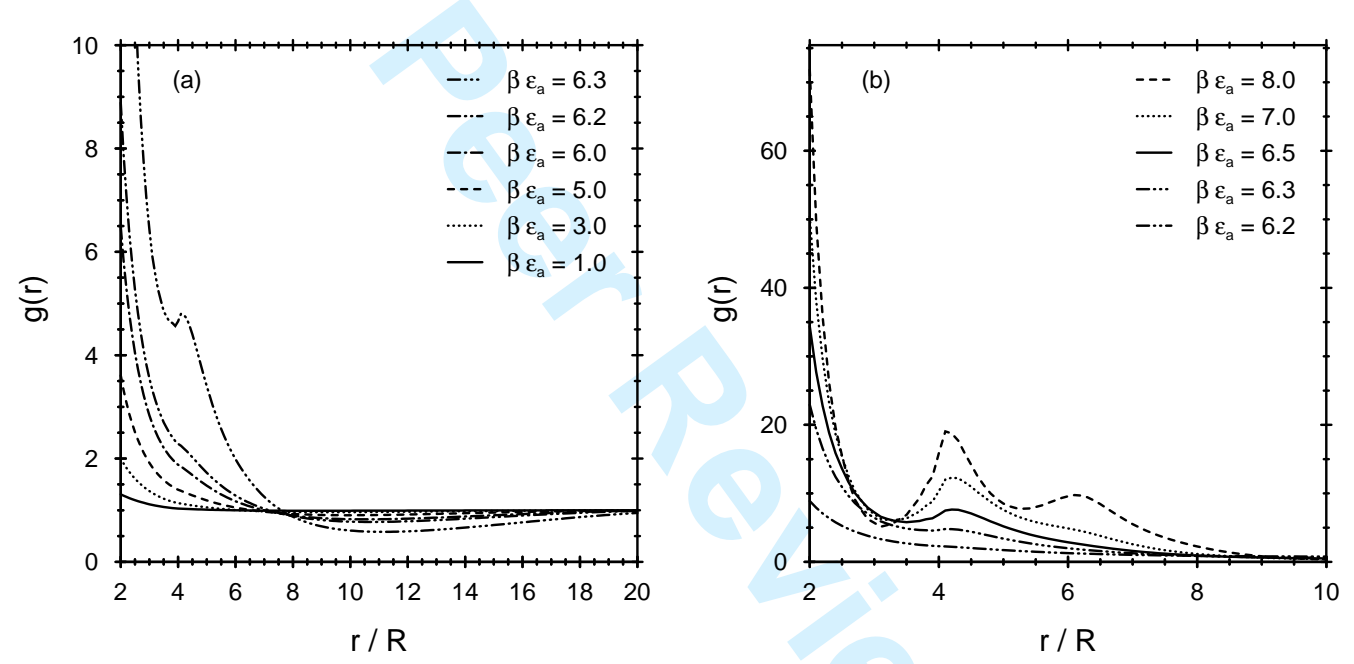

Figure 1. The pair distribution function $g(r)$ at high (a) and at low (b) temperatures for a fluid with a bulk packing fraction of $\eta=0.05$. As the temperature is decreased, the value of $\beta \varepsilon_{a}$ increases. Note that between $\beta \varepsilon_{a}=6.2$ and 6.3, i.e. when the temperature is reduced slightly, there seems to be a jump in $g(r)$ as a peak develops near $r / R=4.2$. This apparent jump is shown both in (a) and (b). At low temperatures, (b), the short-ranged structure in $g(r)$ indicates the formation of clusters.

We begin with the study of the temperature dependence of the pair distribution function $g(r)$ of a fluid with competing interactions for a reservoir packing fraction of $\eta=\rho_{\text {bulk }} R^{2} \pi=0.05$. At such a low value of $\eta$, a fluid of hard disks shows very little structure. However at such a low packing fraction in the present model fluid might be interesting for the following reason. For the parameters chosen in this work, the maximum of the soft potential occurs at a distance of roughly $r_{\max } \approx$ $5.822 R$. If the density is set so that there is in average one disk per area of a circle with radius $r_{\max }$ then one obtains a density of $\rho_{\max }=1 /\left(r_{\max }^{2} \pi\right)$ or an equivalent packing fraction of $\eta_{\max }=\rho_{\max } R^{2} \pi=1 /(5.822)^{2} \approx 0.0295$. In such a dilute system the attractive wells of particles do not have to overlap and an interesting competition between the long ranged repulsion and the attraction at small separation, or a competition between energy and entropy, can be expected.

Within DFT the pair distribution function can be computed in two different ways. In the first route, one generates the direct pair correlation function $c^{(2)}(r)$ 


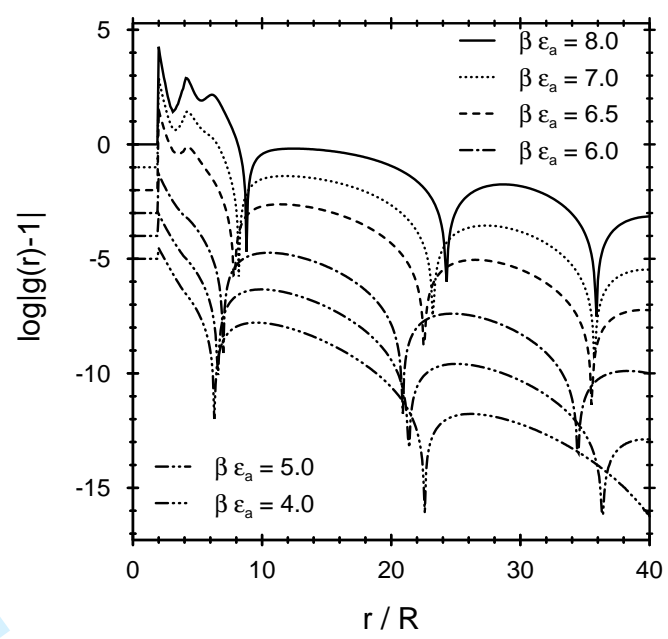

Figure 2. The decay of the pair distribution function $g(r)$ at various temperatures is highlighted by plotting the modulus of $g(r)-1$ logarithmically. For reasons of clarity the different curves are shifted along the y-axes. The asymptotic decay, $r \rightarrow \infty$, reflects the length-scale of the repulsive tail of the interaction. For values of $\beta \varepsilon_{a} \geq 6.3$ (see Fig. 1 and text) the onset of cluster formation can be seen for short distances.

from two derivative of the the excess free energy functional $[14,15]$ which can be inputted into the Ornstein-Zernike equation [1]. The second route, which is the employed here, makes use of the test-particle limit, in which one fluid particle is kept at a fixed position and is thereby turned into an external potential for the rest of the fluid: $V_{\text {ext }}(r)=V(r)$ with $V(r)$ given by (1). The density profile $\rho(r)$ of the fluid subjected to the (radial symmetric) external field of the test particle is obtained by minimising the functional (3). The pair distribution function is this density profile divided by the bulk density, i.e. $g(r)=\rho(r) / \rho_{b u l k}$.

An example for the bulk pair distribution function obtained by the test particle approach for various temperatures is shown in Fig. 1. For high temperatures, e.g. $\beta \varepsilon_{a}=1.0$ in Fig. 1(a) the resulting $g(r)$ shows relatively little structure, and is similar to its hard-disk counterpart, which can be obtained by taking the limit $T \rightarrow \infty$ or $\beta \varepsilon_{a} \rightarrow 0$. As the temperature is reduced the pair distribution functions displays more structure, resulting in a higher contact value of $g(r=2 R)$ and a rather large range of distances in which $g(r)$ deviates significantly from unity.

As the temperature is further decreased the strength of $\beta \varepsilon_{a}$ continues to increase and as it reaches a value between $\beta \varepsilon_{a}=6.2$ and 6.3 there seems to be a jump in $g(r)$ - see Fig. 1(a). In order to show the structure of the pair distribution function at high and at low temperatures and to highlight the rapid change in the pair distribution function, $g(r)$ for the values $\beta \varepsilon_{a}=6.2$ and 6.3 is shown both in part (a) and (b) of Fig. 1. Note that a jump in $g(r)$ would indicate a phase transition. We have confirmed that for values of $\beta \varepsilon_{a}$ between 6.2 and 6.3 the radial distribution function changes rapidly, but continuously. At lower temperatures the pair distribution function starts to show the onset of small clusters of a high density, as indicated by regions close to contact in which $g(r)$ is significantly larger than 1 .

To highlight the decay of the pair distribution function, we plot in Fig. 2 the modulus of $g(r)-1$ logarithmically for various temperatures. For reasons of clarity the curves are shifted along the y-axes. Asymptotically there are oscillations in $g(r)$, which reflect the length-scale of the repulsive tail of the inter-particle interaction. At smaller separations, the onset of cluster formation can be seen clearly for values of $\beta \varepsilon_{a} \geq 6.3$ - see also Fig. 1(b).

By constructions the thermodynamics of the current system in a homogeneous bulk phase (within first-order thermodynamic perturbation theory) is the same as 
that of the hard-disk reference fluid, therefore a macroscopic phase separation into a low density gas and a high density liquid is not possible. However, the formation of clusters, i.e. a micro phase separation into islands of high density in a sea of a low density fluid is possible - in fact the potential, Eq. (2) was introduced in order to study cluster formation $[2,3]$. The behaviour of $g(r)$ shown in Fig. 1 is similar to the results found by Imperio and Reatto [5] in simulation studies of the same model system.

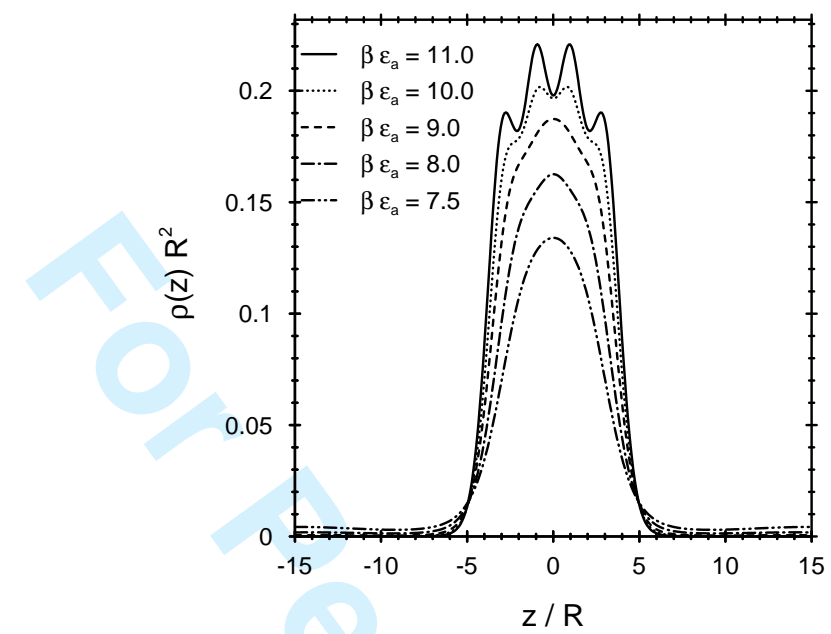

Figure 3. At sufficiently low temperatures a bulk system (without any external field) can form periodic lamellar structures in which high density films are separated by low density regions. The total grand potential of the system in such an inhomogeneous configuration is lower than this of a homogeneous bulk fluid. As the temperature decreases, and $\beta \varepsilon_{a}$ increases, the density in the dense film increases and oscillatory structures develop. For clarity the centre of each high density film is shifted to $z=0$ and roughly one period, which depends on temperature, is shown. Note that the lamellar structure corresponding to $\beta \varepsilon_{a}=7.5$ is slightly meta-stable as compared to a homogeneous bulk fluid, as can be seen from the grand potential shown in Fig. 4.

The particular behaviour of $g(r)$ for small values of $r$ indicates that it should be possible for the system to develop an inhomogeneous density distribution $\rho(\mathbf{r})$ even in the absence of an external potential. If the temperature is sufficiently low then the depth of the attractive well is deep enough to allow the system to lower its overall grand potential by the formation of an inhomogeneous structure. It is at these low temperatures where the competition between the attractive and repulsive part of the interaction results in a clear competition between energy and entropy and the behaviour of the system is particularly rich.

As a proof of principle we consider in this work the low temperature formation of lamellar films, i.e. periodic arrays of high density fluids, separated by low density regions. We assume translational invariance in the $x$-direction and consider density profiles that depend only on $z$. A set of typical structures for a reservoir packing fraction of $\eta=0.05$ is shown in Fig. 3. For reasons of clarity the centre of the high density films for each temperature is shifted to $z=0$.

With decreasing temperature the density in the film around $z=0$ increases and reaches such high values that oscillatory structures due to packing effects become clearly visible. Note that in order to describe these packing effects properly within DFT, the hard-core repulsion must be treated by weighted density approximation such as fundamental measure theory [16-19], while the presence of lamellar structures can also be found within a local density approximation approach for the hard-disk functional (for a slightly changed interaction potential) [12].

The width of the high density film and the length of a full period, i.e. the distance between two neighbouring films, depends on the temperature. In order to find the 


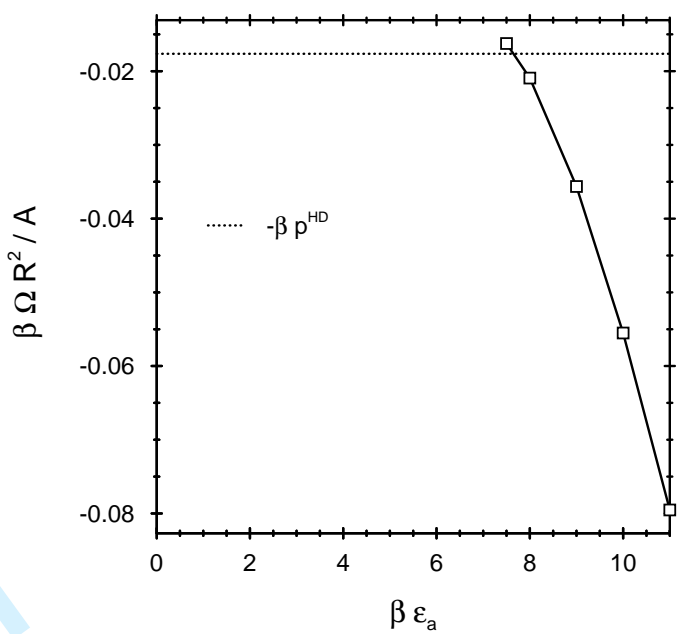

Figure 4. The grand potential density $\beta \Omega / A$ of a bulk system at $\eta=0.05$. The dotted line denotes the grand potential density of a homogeneous bulk fluid at $\eta=0.05$, for which $\beta \Omega / A=-\beta p^{H D}$, while the full line denotes that of a periodic lamellar structure. The symbols correspond to the values of the grand potential density of the density profiles shown in Fig. 3. Note that the grand potential density of the lamellar structure for $\beta \varepsilon_{a}=7.5$ is higher than that of a homogeneous bulk fluid at the same temperature, indicating that the inhomogeneous structure is meta-stable at this state point. Stable lamellar films exist for $\beta \varepsilon_{a} \gtrsim 7.67$.

most stable lamellar structure, the density functional must be minimised w.r.t. the size of the periodic box employed in this calculation. This is done in two steps. In the first step we fix the size $h_{z}$ of the periodic box in $z$ direction and minimise the functional. This results in an inhomogeneous density profile $\rho\left(z ; h_{z}\right)$, which depends parametrically on $h_{z}$. Also the grand potential density depends on the parameter $h_{z}$ via $\beta \Omega\left(h_{z}\right) / A=\beta \Omega\left[\rho\left(z ; h_{z}\right)\right] / A$. In the second step the grand potential density is minimised w.r.t. $h_{z}$. As a result of this second minimisation Fig. 3 shows roughly, but not precisely, one period of the lamellar films; with decreasing temperature the period of the structure decreases.

In Fig. 4 we show the grand potential density $\beta \Omega / A$ minimised w.r.t. $h_{z}$ and corresponding to the density profiles $\rho(z)$ shown in Fig. 3 (full line and symbols) and compare it to that of a homogeneous bulk fluid, Eqs. (12) and (13), (dotted line). As can be seen, the inhomogeneous lamellar structure for $\beta \varepsilon_{a}=7.5$ is metastable compared to a homogeneous bulk fluid, but for temperatures lower than this, the grand potential density rapidly decreases, showing that the system can reduce the total grand potential by the formation of inhomogeneous structures. This is in line with the finding that the radial distribution function shown in Fig. 1 displays a tendency of the system for micro phase-separation (cluster formation) at low temperatures. However, the temperatures at which $g(r)$ displays a rapid change (between $\beta \varepsilon_{a}=6.2$ and 6.3) and that at which the bulk forms a stable lamellar structure at $\beta \varepsilon_{a} \approx 7.67$ are clearly different. This finding indicates that it is easier for the system at this state point to form circular clusters than translational invariant lamellar films.

If the constraint of translational invariance in one direction is lifted, a zoo of rich inhomogeneous bulk structures can be found [12]. While we have confirmed this for a few examples of this system a detailed study of these structures is beyond the scope of the present study. 


\section{A Single Hard Wall}

If a homogeneous fluid is subjected to an external potential $V_{\text {ext }}(\mathbf{r})$, in general it develops an inhomogeneous density distribution $\rho(\mathbf{r})$ with the same symmetry as the external potential. Here we study the influence of a single planar hard wall on the structure of a fluid with competing interactions. Note that in the case of a homogeneous bulk phase, far away from the wall, the density profile close to the hard wall must satisfy the contact theorem which states that the contact density $\rho\left(z=0^{+}\right)=\lim _{\epsilon \rightarrow 0} \rho(z=\epsilon)$ is proportional to the bulk pressure

$$
\rho\left(z=0^{+}\right)=\beta p
$$

which for the present model coincides with the pressure $p^{H D}$ of the hard-disk reference fluid. The contact theorem is obeyed by profiles from the functional (6) $[17]$.
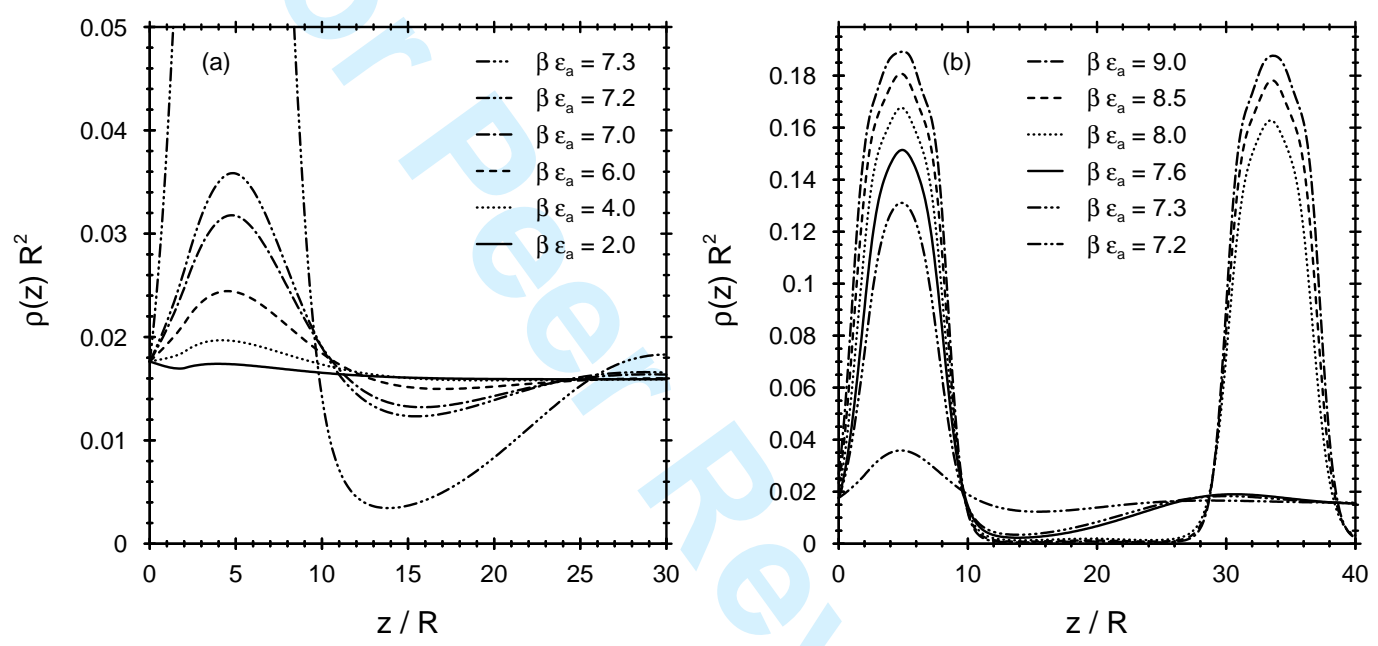

Figure 5. The density profiles of a fluid with competing interactions at a planar hard wall for high (a) and low (b) temperatures, respectively. The bulk density $\rho(\infty) R^{2}=0.05 / p i \approx=0.0159$. The contact density $(z=0$ in this plot) is fixed to be the bulk pressure, which coincides with the hard-disk pressure as long as the bulk phase, far away from the wall, is a homogeneous fluid. Note that between $\beta \varepsilon_{a}=7.2$ and 7.3 a clear jump occurs in the density peak close to the wall. In order to highlight the magnitude of the jump, the density profiles corresponding to $\beta \varepsilon_{a}=7.2$ and 7.3 are shown both in (a) and (b). For $\beta \varepsilon_{a}>7.64$ the density profile away from the wall displays a lamellar structure.

In Fig. 5 we show the density profiles $\rho(z)$ for a reservoir packing fraction of $\eta=0.05$ at various temperatures. For sufficiently high temperatures, i.e. for $\beta \varepsilon_{a}<$ 2.0, one can observe a profile that resembles that of a dilute hard-disk fluid close to a planar hard wall: at contact the satisfies (14) and away from the wall the density decays in a damped oscillatory fashion towards the bulk density. Since the reservoir packing fraction is rather small, the decay of the density profile at high temperatures towards its bulk value takes place within the range of a few particle radii. Note that for this value of the packing fraction the contact density is fixed via (12) and (14) to be $\rho\left(0^{+}\right) R^{2}=\rho_{\text {bulk }} R^{2} /(1-\eta)^{2}=\eta /\left(\pi(1-\eta)^{2}\right)=0.017635$. We find the contact theorem to be satisfied by our DFT results, as can be seen by the common density at $z=0$ in Fig. 5(a) and (b), except for profile with $\beta \varepsilon_{a} \geq 8.0$.

Already at a temperature corresponding to $\beta \varepsilon_{a}=2.0$ the resulting density profile differs significantly from that of hard disks as there is the onset of a density peak near $z=5 R$ forming, which on the scale of Fig. 5(a) is hardly noticeable. This density peak becomes more pronounced as the temperature is reduced and as $\beta \varepsilon_{a}$ 
reaches a value between 7.2 and 7.3 a clear jump from a moderate peak height to a large one can be observed. In order to highlight the amplitude of this jump, the density profiles for $\beta \varepsilon_{a}=7.2$ and 7.3 are shown in both parts of Fig. 5 .

At first sight the density profiles for temperatures slightly below the temperature of the jump seem to be that of the high density part of a lamellar structure - see Fig. 3 - there is a clear and important difference. For temperatures above that corresponding to $\beta \varepsilon_{a}=7.64$ the density profiles still decay towards a homogeneous bulk fluid. This in turn implies that above this temperature the contact theorem must be satisfied. In other words, the contact density of all density profiles shown in Fig. 5, except for those corresponding to $\beta \varepsilon_{a} \geq 8.0$, is the same and is given by Eq. (14).

Eventually the temperature is low enough for an inhomogeneous structure forming away from the wall that does not decay any longer towards a homogeneous fluid. In that case the wall theorem, Eq. (14), no longer applies and the density at contact differs from $\beta p^{H D}$.

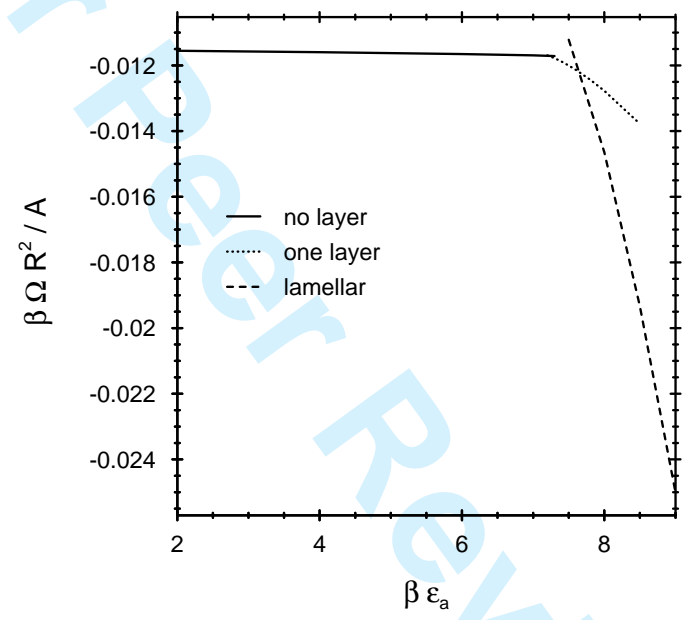

Figure 6. The grand potential density $\beta \Omega / a$ of a fluid with $\eta=0.05$ in contact with a single hard wall. There are three branches. The full line corresponds to density profiles without a high density layer at the wall. This brach intersect with the second branch (dotted line) at $\beta \varepsilon_{a} \approx 7.22$. The second branch corresponds to a fluid with one high density layer at the wall. The third branch (dashed lines) corresponds to a lamellar structure in contact with the wall. The second and third branch of the grand potential density intersect at $\beta \varepsilon_{a} \approx 7.64$.

In order to understand the behaviour of the density profiles better, we show in Fig. 6 the grand potential density of a fluid with $\eta=0.05$ in contact with a planar hard wall. We find three distinct branches of the grand potential density. The first branch (full line in Fig. 6) corresponds to density profiles with no high density layers close to the wall. At $\beta \varepsilon_{a} \approx 7.22$ this first branch intersect with a second branch of the grand potential density, which corresponds to density profiles with a single high density layer (dotted line in Fig. 6). Although not visible on the scale of Fig. 6, DFT predicts a sharp, first-order phase transition with a small range of meta-stable states, between these two states. In a real system this transition would be rounded and smooth due to thermal fluctuations. At $\beta \varepsilon_{a} \approx 7.64$ the second branch of the grand potential density intersects with the third branch (dashed line in Fig. 6), which corresponds to a lamellar structure in contact with the wall. This transition shows hysteresis effects with large regions of possible meta-stable states and should be a true first-order phase transition. 


\section{An Infinite Slit Pore}

If a fluid is confined by a slit pore of two parallel and planar hard walls of infinite lateral extension $L_{x} \rightarrow \infty$, separated by a width $L$, then the contact theorem (14) no longer applies. Only if the walls are infinitely separated, so that the system actually consists of two independent walls, will the contact theorem be valid. For practical purposes $L \rightarrow \infty$ means a wall separation large compared to the bulk correlation length. Here we fix $L=20 R$, which is large compared the bulk correlation length of a hard-disk fluid at the reservoir packing fraction $\eta=0.05$ considered here, but is comparable to the length of one period of a lamellar structure, which is roughly $30 R$ as can be seen from Fig. 3. It follows that the contact density in the slit at high temperatures should be close to $\beta p^{H D}$.

In Fig. 7 we show the density profiles $\rho(z)$ in a slit of with a width $L=20 R$ for a reservoir packing fraction of $\eta=0.05$ for high (a) and low (b) temperatures, respectively. At high temperatures, Fig. 7(a), we find as expected, a contact density close to the hard-disk pressure multiplied by $\beta$. As the temperature decreases a density peak develops in the middle of the slit and the contact density at decreases. For $\beta \varepsilon_{a}$ between 7.2 and 7.3 the density peak in the middle of the slit pore jumps to a high value and the contact density to a rather low one. For clarity the density profiles for $\beta \varepsilon_{a}=7.2$ and 7.3 are shown in both in parts of Fig. 7.

As the temperature is further decreased the high density peak in the middle of the slit increases and becomes similar, but not identical, to that of the lamellar structure, shown in Fig. 3.
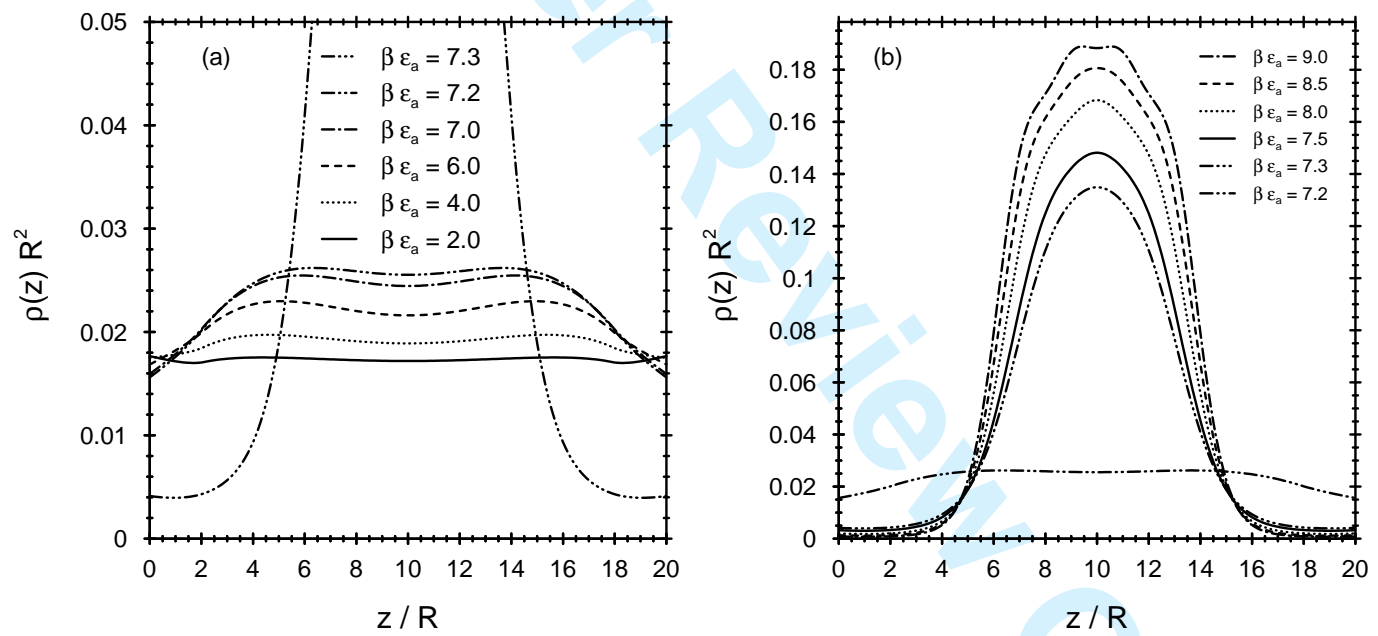

Figure 7. Density profiles of a fluid with competing interactions inside a slit of two parallel hard walls at a separation of $L=20 R$ at high (a) and low (b) temperatures respectively. As the temperature decreases the density of the fluid in the central part of the slit increases smoothly. Between $\beta \varepsilon_{a} 7.3$ and 7.4 the density jumps. In order to highlight the magnitude of the jump, the density profiles corresponding to $\beta \varepsilon_{a}=7.3$ and 7.4 are shown in (a) and (b). The width of the slit is somewhat smaller than the width of a single period of the bulk lamellar structures (see Fig. 3).

The grand potential of the system confined in a slit pore divided by the length $L_{x}$ of the slit in the $x$-direction is shown in Fig. 8 and is numerically similar to the grand potential density of a lamellar phase, shown in Fig. 4, if the result for the slit is divided by the slit width $L=20 R$. There are two branches of the grand potential, one for the low density and one for the high density phase. These two branches intersect at $\beta \varepsilon_{a} \approx 7.24$. Around the transition temperature meta-stable structures can exist.

Note that the transition from a low density phase to a high density phase ap- 


\section{Page 11 of 12

pears to occur in the slit pore at a lower value of $\beta \varepsilon_{a}$, corresponding to a higher temperature, than in the bulk. This shift in transition for a fluid with competing interactions can be viewed as a transition analogous to capillary condensation in a simple fluid.

In a true system the sharp transition depicted in Fig. 8 would be rounded and smooth, as thermal fluctuation in this effective one-dimensional system would be strong.

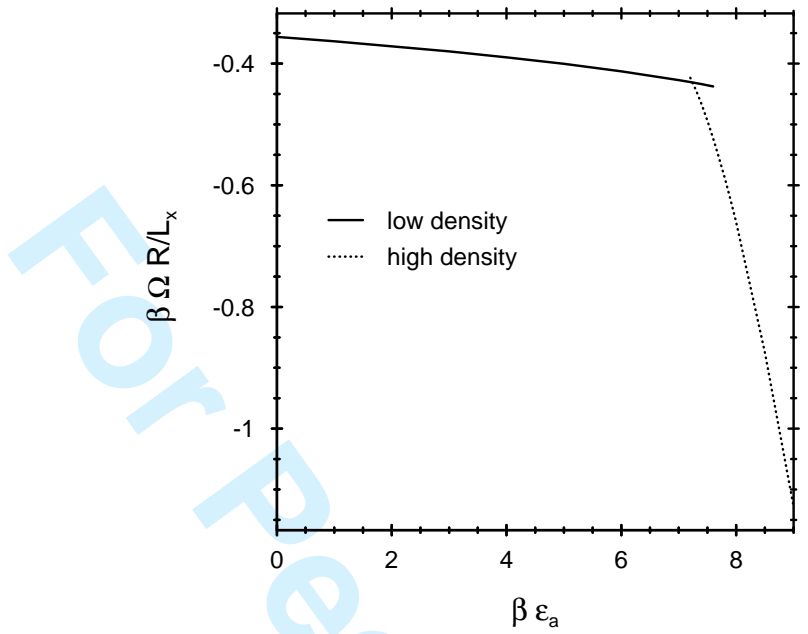

Figure 8. The grand potential $\beta \Omega$ divided by the length of the slit $L_{x}$. For high temperatures the average density of the fluid inside the slit is low, while it is high at low temperatures. There are two branches of the grand potential, which intersects at a transition temperature corresponding to $\beta \varepsilon_{a} \approx 7.24$. Slightly below the transition temperature a meta-stable low density state can be observed, and slightly above the transition point a meta-stable high density state can be present.

As mentioned in Sec. 3, it is possible to find a variety of more complex structures confined in a slit pore $[6,12]$. While in principle it is possible to study these structures with the present approach, it is beyond the scope of this study to do so.

\section{Discussion}

We have studied the fluid behaviour of a two dimensional system of particles with competing interactions within the framework of DFT. The interaction potential, Eqs. (1) and (2), was accounted for by a reference system of hard disks, treated within FMT [16-19], together with a first order perturbation theory for the soft interaction.

The model interaction, Eq. (2), was introduced by Sear et al. $[2,3]$ to study cluster formation of nanoparticles (quantum dots) at a water-air interface. Reatto has recognised the rich behaviour of systems with competing interactions and he and co-workers have studied in detail the equilibrium properties of a two dimensional system with the pair interaction given by Eqs. (1) and (2) [4-6] and by a slightly different interaction $[8-10]$ in three dimensions.

This study was inspired by the findings and insights of Reatto and co-workers [4-6]. The results found here are in broad agreement with the results of the simulation studies by Imperio and Reatto. We believe that by using the framework of DFT we have added a slightly different perspective on some of the equilibrium properties of systems with competing interactions. DFT allows to study the structure (density profile) and the thermodynamics (grand potential) of a system on 
equal footing. Therefore DFT allows one directly to study the range of stability of certain structures, as in Figs. 4 and 8.

Here we have reported results for one, rather low, value of the reservoir packing fraction $\eta=0.05$. For higher values of $\eta$ we find a similar behaviour in many respects. Details change, however. For example, we find that some jumps in density profiles, reported here, become continuous transitions. The meta-stable states found for $\eta=0.05$ seem to disappear at higher values of $\eta$ and the transition from the low density branch of the grand potential to the high density one seems to be smooth. This observation is consistent with results reported in Ref. [10] for a three-dimensional system with slightly different competing interaction using a order parameter theory.

DFT was found to be a powerful tool to study two dimensional systems with competing interactions. The behaviour of this system is already rich and interesting in very simple external potentials, such as at a single planar hard wall. The behaviour becomes even richer, if a more complex external field is applied [4].

\section{Acknowledgments}

This work is dedicated to Luciano Reatto, who through his work and insights inspired many researchers, including me, in the liquid state community. Throughout the work on this manuscript I had the pleasure to discuss the results presented here and their implications with Michael Klatt, for which I am very grateful. Finally, I want to thank Bob Evans for helpful discussions and comments on the manuscript.

\section{References}

[1] J. P. Hansen and I. R. McDonald, Theory of simple liquids (Academic Press, London) (1986).

[2] R. Sear and W. M. Gelbart, J. Chem. Phys. 1104582 (1998).

[3]R. P. Sear, S.-W. Chung, G. Markovich, W. M. Gelbart, and J. R. Heath, Phys. Rev. E 59, R6255 (1999).

[4]A. Imperio and L. Reatto, J. Phys.: Condens. Matter 16, S3769 (2004).

[5]A. Imperio and L. Reatto, J. Chem. Phys. 124, 164712 (2006).

[6] A. Imperio and L. Reatto, Phys. Rev. E 76, 040402 (2007).

[7]N. Destainville, Phys. Rev. E 011905 (2008).

[8]D. Pini, G. Jialin, A. Parola, and L. Reatto, Chem. Phys. Lett. 327, 209 (2000).

[9] A. J. Archer, D. Pini, R. Evans, and L. Reatto, J. Chem. Phys. 126, 014104 (2007).

[10] A. J. Archer, C. Ionescu, D. Pini, and L. Reatto, J. Phys.: Condens. Matter 20, 415106 (2008).

[11]A. J. Archer, and N. Wilding, Phys. Rev. E 76, 031501 (2007).

[12]A. J. Archer, Phys. Rev. E 78, 031402 (2008).

[13]Z. Li and J. Wu, J. Chem. Phys. 130, 165102 (2009).

[14]R. Evans, Adv. Phys. 28, 143 (1979).

[15]R. Evans, in Fundamentals of In homogeneous Fluids, edited by D. Henderson (Marcel Dekker, New York, 1992) p. 85.

[16]Y. Rosenfeld, Phys. Rev. A 42, 5978 (1990).

[17]R. Roth, K. Mecke, and M. Oettel, submitted (2011).

[18] Y. Rosenfeld, Phys Rev. Lett. 63, 980 (1989).

[19]R. Roth, J. Phys.: Condens. Matter 22, 063102 (2010). 